\title{
COVID-19 and Toll-like Receptor 4 (TLR4): SARS-CoV-2 may activate TLR4 to increase ACE2 expression for entry and cause abnormal inflammatory signalling: A Cardio-Respiratory focus.
}

\author{
Dr Richard Heads ${ }^{1}$ \\ ${ }^{1}$ King's College
}

September 14, 2020

\begin{abstract}
Causes of mortality from COVID-19 include respiratory failure, heart failure, and sepsis/multi-organ failure. TLR4 is an innate immune receptor on the cell surface that recognizes Pathogen Associated Molecular Patterns (PAMPs) including viral proteins and triggers the production of type I interferons and proinflammatory cytokines to combat infection. It is expressed on both immune cells and tissue-resident cells. ACE2, the reported entry receptor for SARS-CoV-2, is only present on $1-2 \%$ of the cells in the lungs or has a low pulmonary expression, and recently the spike protein has been proposed to have the strongest protein-protein interaction with TLR4. Here we review and connect evidence for SARS-CoV-1 and SARS-CoV2 having direct and indirect binding to TLR4, together with other viral precedents, which when combined shed light on the COVID-19 pathophysiological puzzle. We propose a model in which the SARS-CoV-2 spike glycoprotein binds TLR4 and activates TLR4 signalling to increase cell surface expression of ACE2 (since it is a human interferon-stimulated gene, expressed downstream) in order to enter the cells. This would allow SARS-CoV-2 to infect more cells whether in the lungs or heart. SARS-CoV-2 also destroys the type II alveolar cells that secrete pulmonary surfactants, which normally decrease the air/tissue surface tension and block TLR4 in the lungs. This not only leads to a sharp reduction in pulmonary surfactants, breathing difficulties and ARDS, thereby explaining the symptoms, but also exposure of TLR4 on the lung cells allowing more viral infection and inflammation. Furthermore, SARS-CoV-2 is likely causing myocarditis and multiple-organ injury by triggering excessive TLR4 activation and aberrant TLR4 signalling; favouring the canonical MyD88- dependent pro-inflammatory pathway, over the alternative TRIF/TRAM-dependent anti-inflammatory and interferon pathway. This may also explain in part, the severe hyperinflammation or "cytokine storm" that occurs in COVID-19 patients. The TLR4 pathway triggered may depend on the cell type and stage of infection. Moreover, Damage Associated Molecular Patterns (DAMPs) released from necrotic cells further activate TLR4 to cause inflammation, and possibly fibrosis. There are also possibilities that SARS-CoV-2 may use TLR4 to enter the cells via internalisation into endosomes in the alternative pathway or activate TLR4 on platelets to cause pro-thrombotic events. A number of clinical trials have been initiated testing the use of pulmonary surfactants in COVID-19 patients, similar to treating Neonatal Respiratory Distress Syndrome. Pulmonary surfactants would prevent alveolar collapse, prevent infection of naïve cells and block TLR4 from the virus and/or DAMPs. TLR4 antagonists have also been trialled in sepsis or in an antiviral context and may be useful in the treatment of COVID-19. As such, we believe that TLR4 contributes significantly to the pathogenesis of SARS-CoV-2 and its over-activation causes a prolonged or excessive innate immune response.
\end{abstract}

\section{Hosted file}

TLR4_COVID_Review_HEADS.pdf available at https://authorea.com/users/357590/articles/480972covid-19-and-toll-like-receptor-4-tlr4-sars-cov-2-may-activate-tlr4-to-increase-ace2expression-for-entry-and-cause-abnormal-inflammatory-signalling-a-cardio-respiratoryfocus 